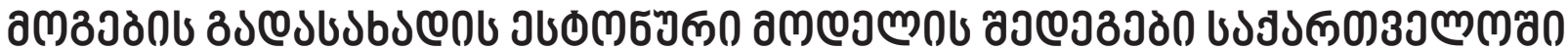

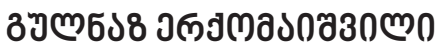

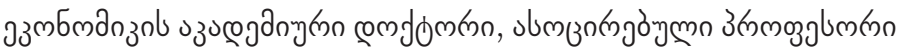

https://doi.org/10.35945/gb.2018.06.023

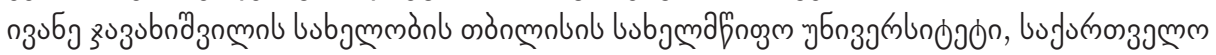

gulnazi.erkomaishvili@tsu.ge

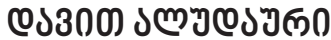

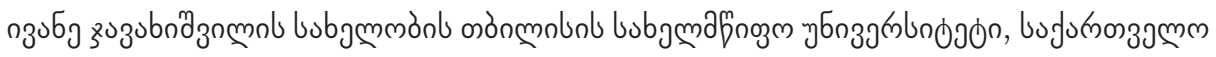

davit.aludauri@gmail.com

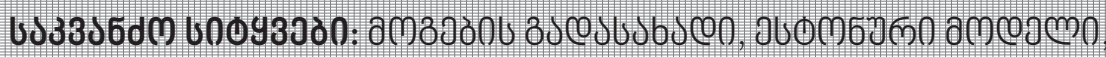

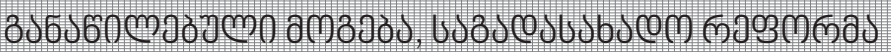

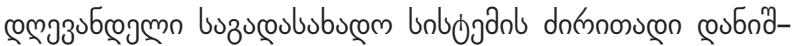

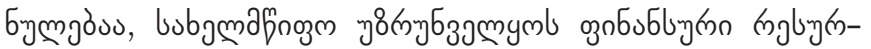

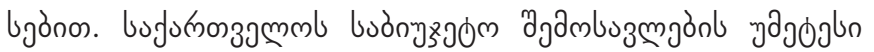

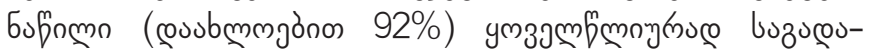

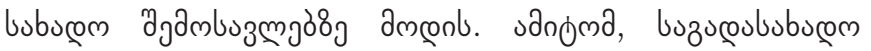

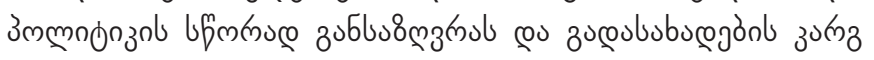

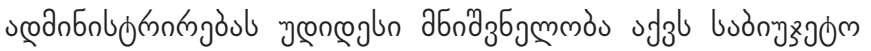

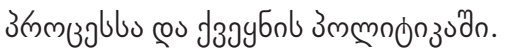

sbumn buzucoububugem lnboganl huamyumnòjò

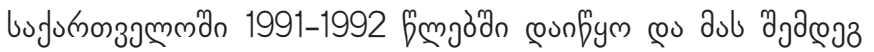

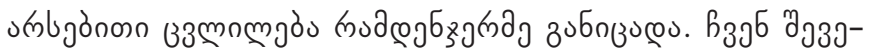

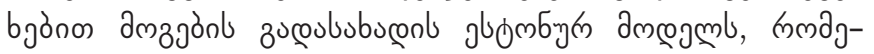

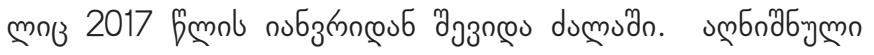

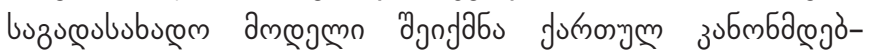

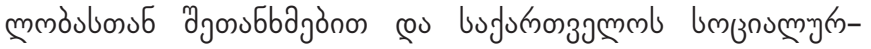

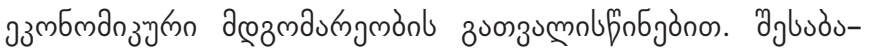

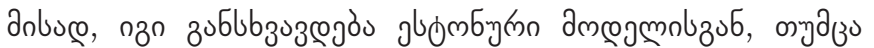

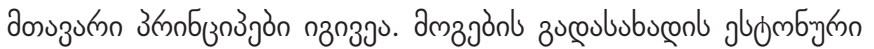

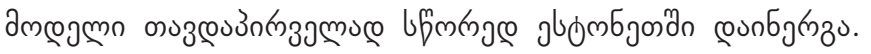

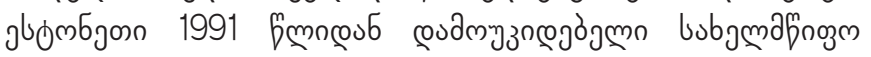

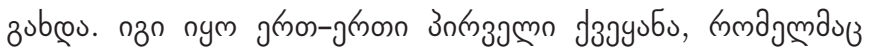

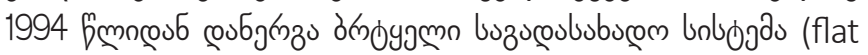

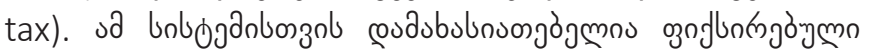

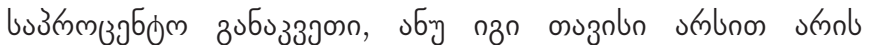

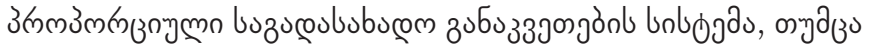

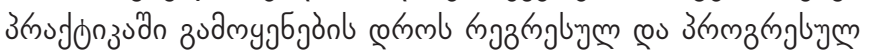

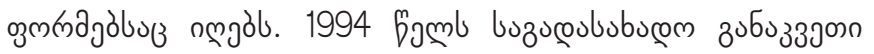

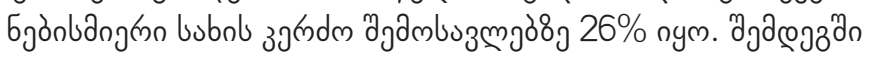

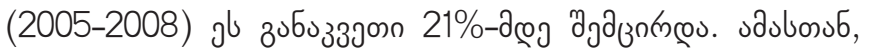

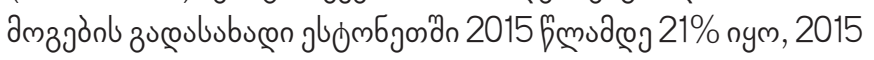

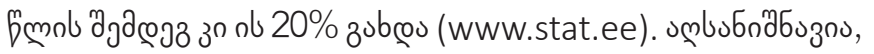

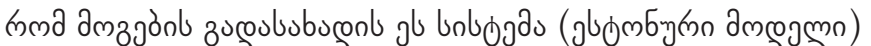

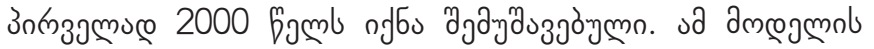

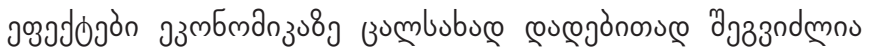

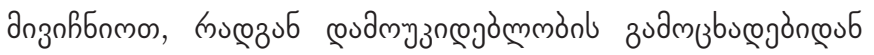

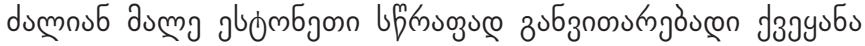

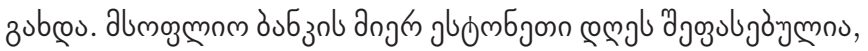

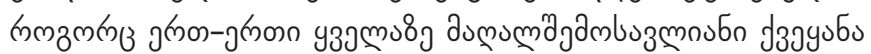

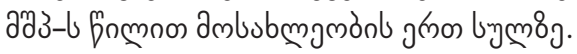

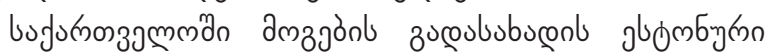

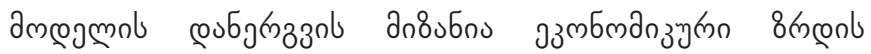

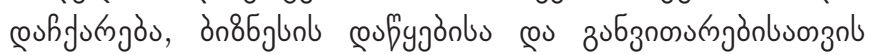

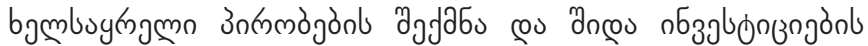

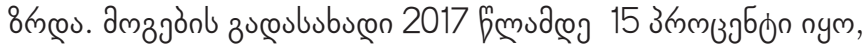

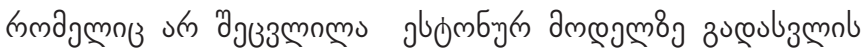

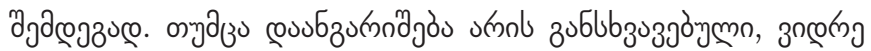

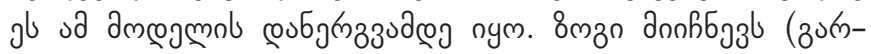

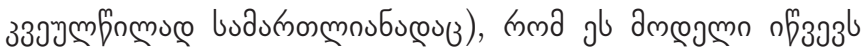

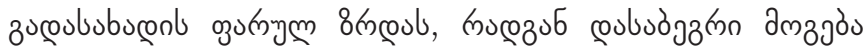

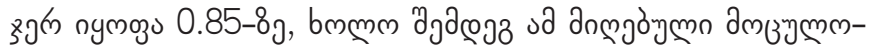

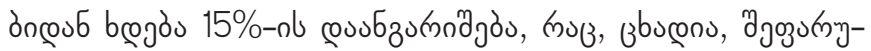

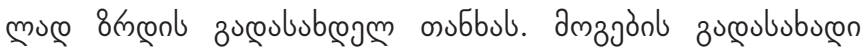

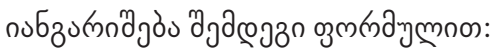

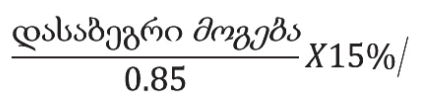

zonjzum, zmazsannl amzjòs smol 1000 mumn. 2017

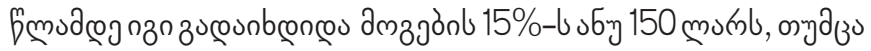

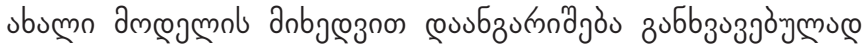

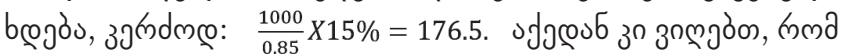

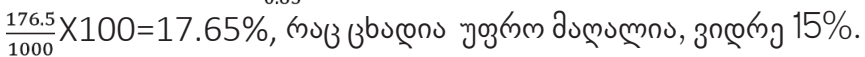

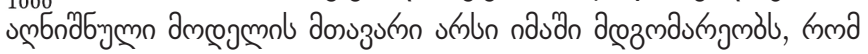

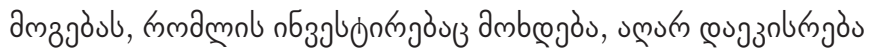

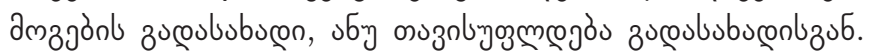

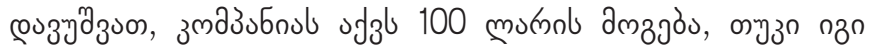

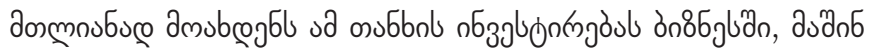

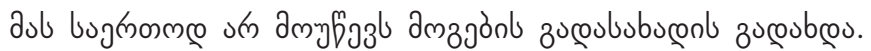

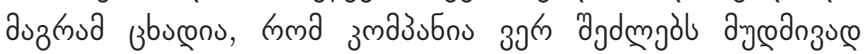

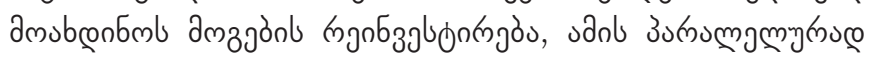




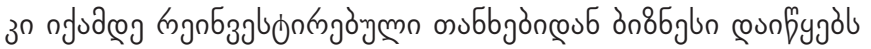

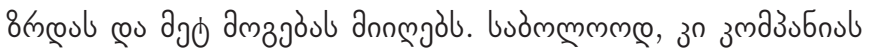
aunб

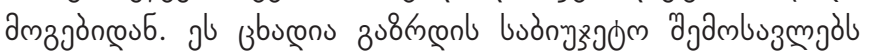

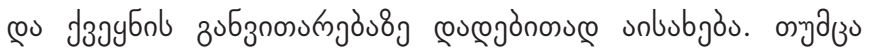

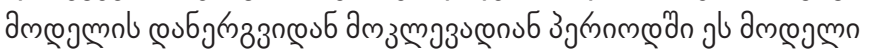

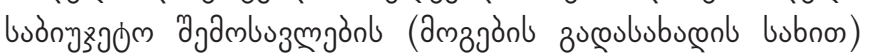

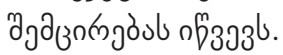

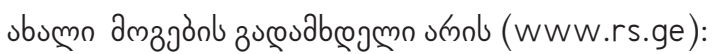

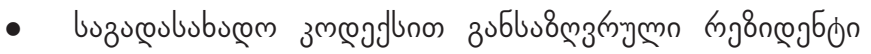

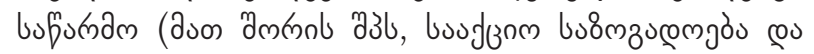

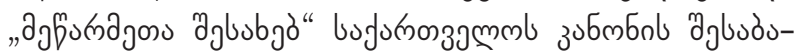

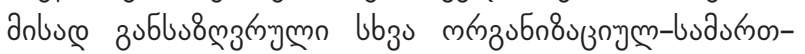

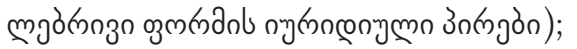

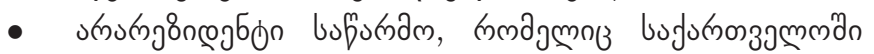

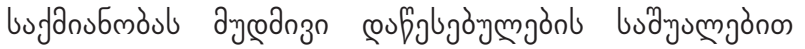

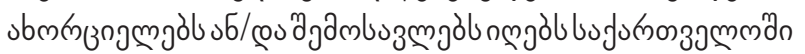

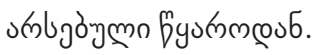

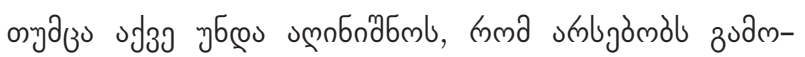

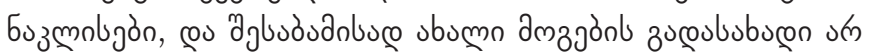
jbjos:

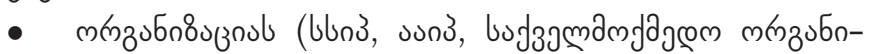
8o(zoul);

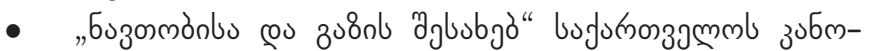

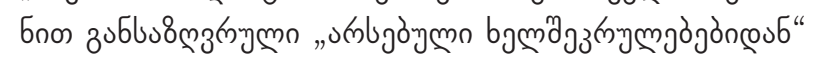

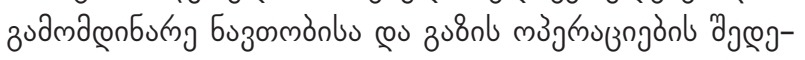

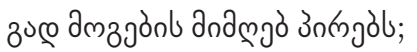

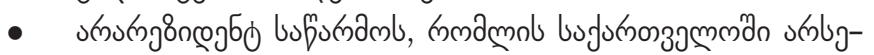

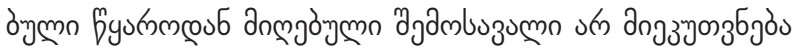
d

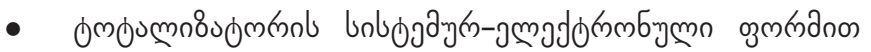

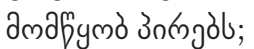

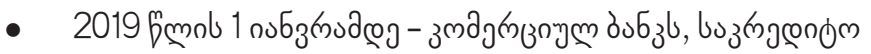

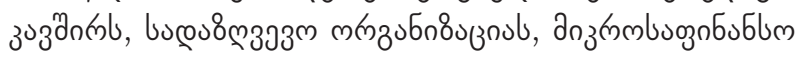

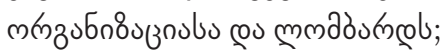

-

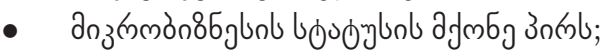

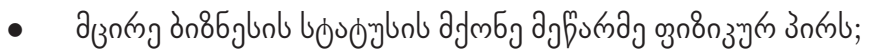

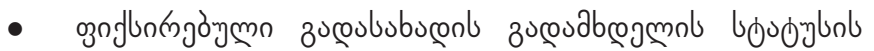
ajmbg 30 的l.

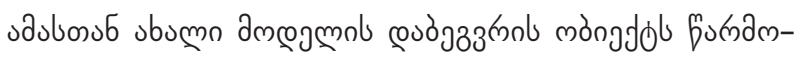
งеgjbl:

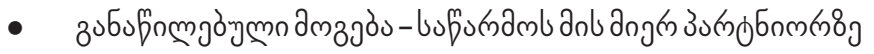

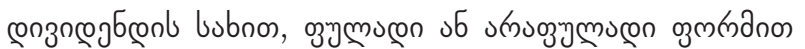

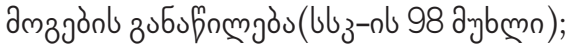

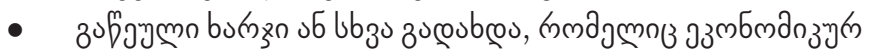

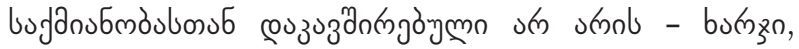

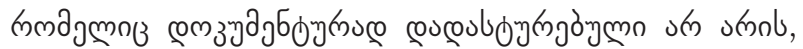

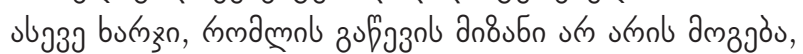

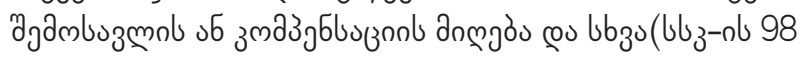
aybmo);

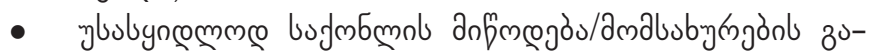

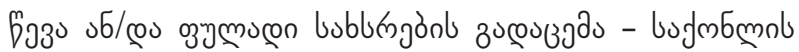

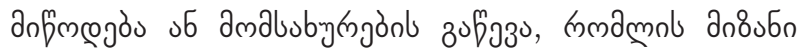

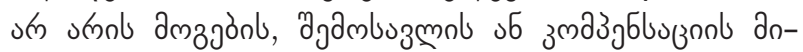

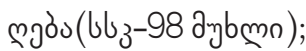

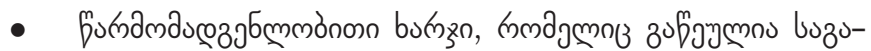

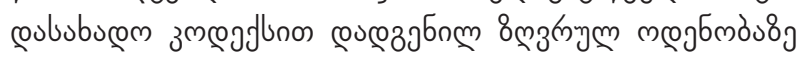

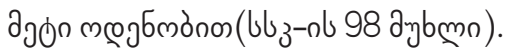

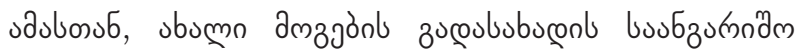

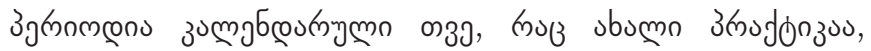

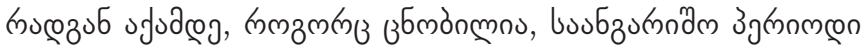

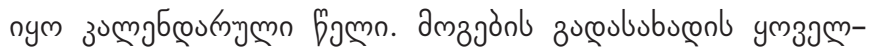

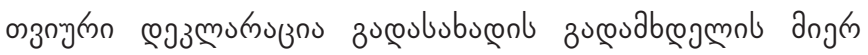

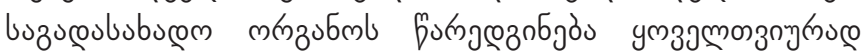

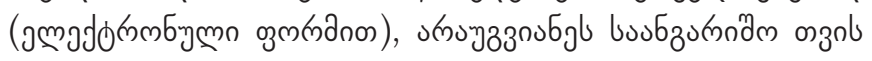

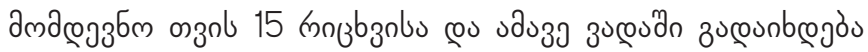

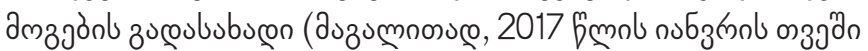

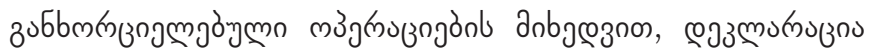

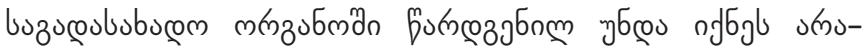

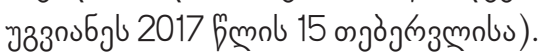

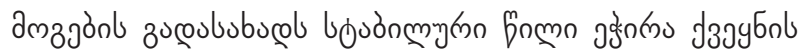

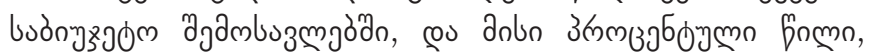

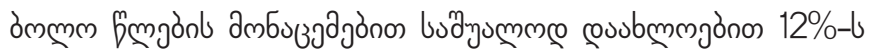

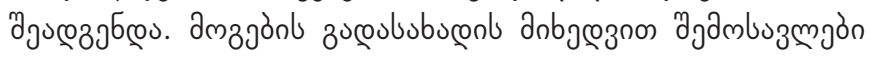

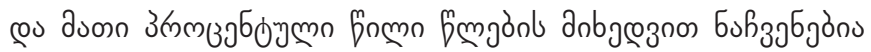

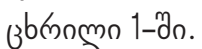

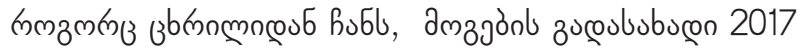

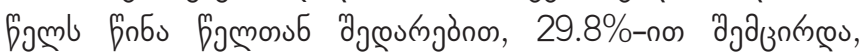

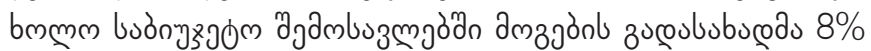

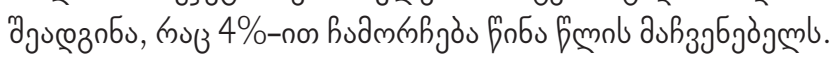

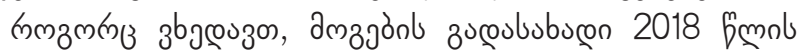

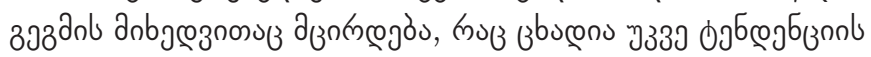

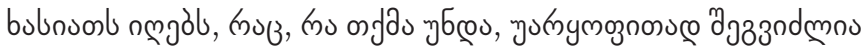

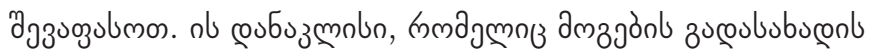

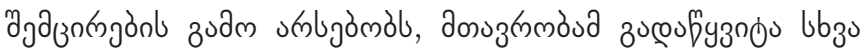

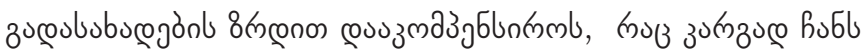

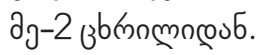

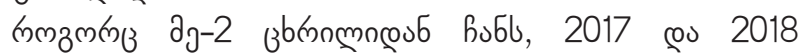

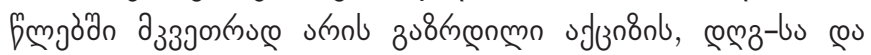

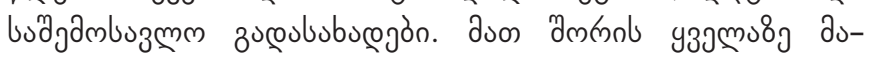

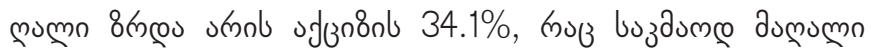

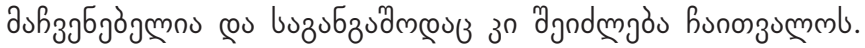

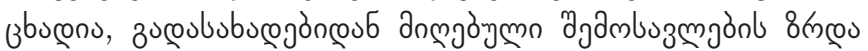




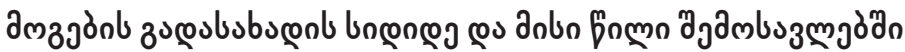

obronmo 1 (www.mof.gov.ge)

\begin{tabular}{|c|c|c|c|c|c|c|c|c|c|c|c|c|}
\hline Бิm๐ & 2007 & 2008 & 2009 & 2010 & 2011 & 2012 & 2013 & 2014 & 2015 & 2016 & $\begin{array}{l}2017 \\
303 \partial\lrcorner\end{array}$ & $\begin{array}{l}2018 \\
30 \partial^{\partial} \mathrm{s}\end{array}$ \\
\hline 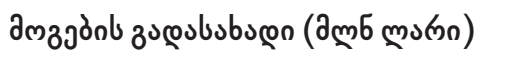 & 555 & 592 & 518 & 576 & 832 & 851 & 807 & 829 & 1025 & 1055 & $\underline{740}$ & $\underline{630}$ \\
\hline 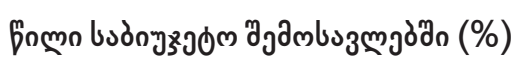 & 12 & 11 & 11 & 11 & 13 & 12 & 12 & 11 & 13 & 12 & 8 & $\underline{6}$ \\
\hline
\end{tabular}

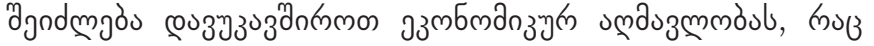

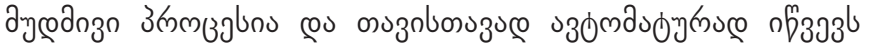

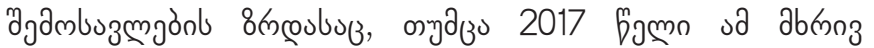

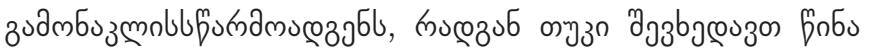

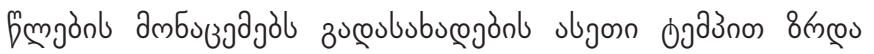

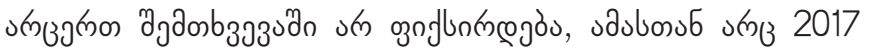

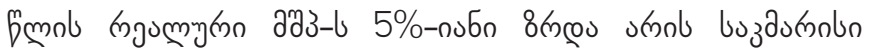

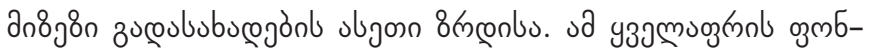

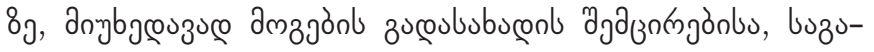

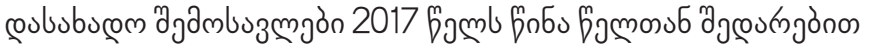

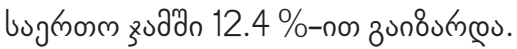

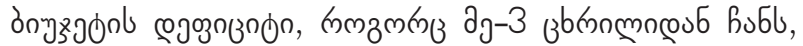

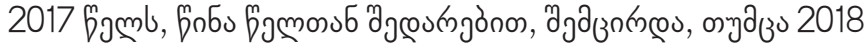

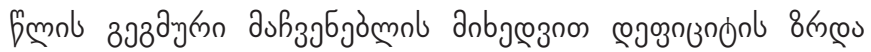

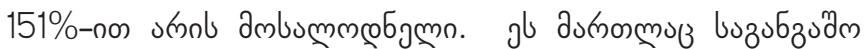
auf3jб

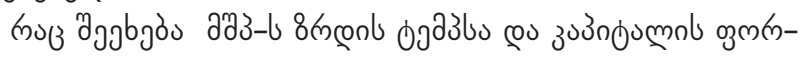

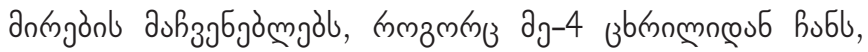

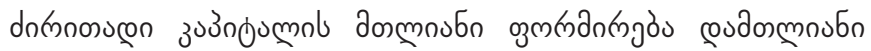

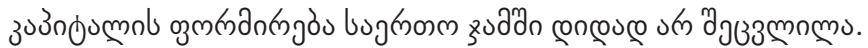

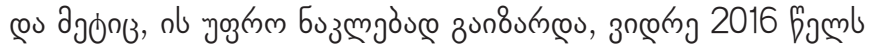

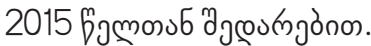

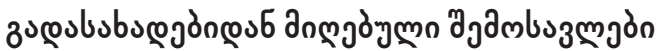

3bronmon 2 (www.mof.gov.ge)

\begin{tabular}{|c|c|c|c|c|}
\hline Бэмू & 2015 & 2016 & $\begin{array}{l}2017 \\
\partial \partial^{2} \partial \Delta\end{array}$ & $\begin{array}{l}2018 \\
3 \partial^{2} \partial^{\prime}\end{array}$ \\
\hline 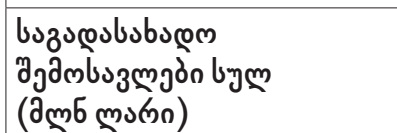 & 7550 & 7987 & 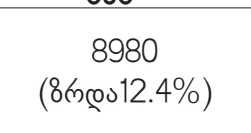 & $\begin{array}{c}9490 \\
\text { (8mஹே 5.7\%) }\end{array}$ \\
\hline 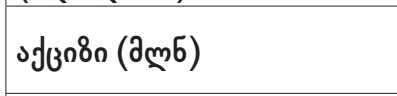 & 871 & 1070 & $\begin{array}{c}1435 \\
(8 \text { m go 34.1\%) }\end{array}$ & $\begin{array}{c}1450 \\
(8 \text { 띠 } 1 \%)\end{array}$ \\
\hline 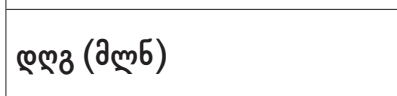 & 3505 & 3286 & $\begin{array}{c}4020 \\
8 \operatorname{mg0}(22.3 \%)\end{array}$ & $\begin{array}{c}4400 \\
(8 \text { mos } 9.4 \%)\end{array}$ \\
\hline 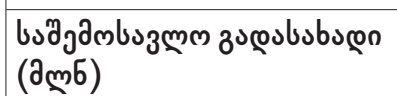 & 2052 & 1978 & $\begin{array}{c}2570 \\
(8 \operatorname{mos} 30 \%)\end{array}$ & $\begin{array}{c}2780 \\
(8 \text { (n) 8\%) }\end{array}$ \\
\hline 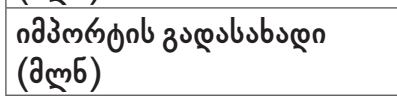 & 69 & 70 & 65 & 60 \\
\hline 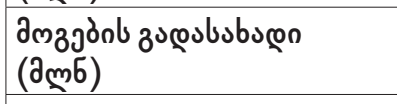 & 1025 & 1055 & 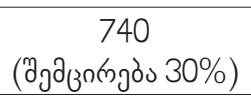 & 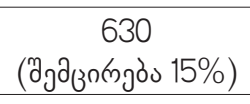 \\
\hline 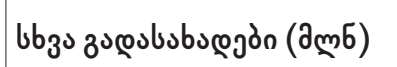 & 27 & 527 & 150 & 170 \\
\hline
\end{tabular}

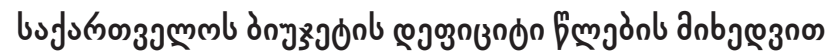

Bbrnnmo 3 (www.mof.gov.ge)

\begin{tabular}{|c|c|c|c|c|c|c|c|}
\hline Бெмूn & 2012 & 2013 & 2014 & 2015 & 2016 & $\begin{array}{l}2017 \\
30 \partial^{2} \Delta\end{array}$ & $\begin{array}{c}2018 \\
30 \partial^{2}\end{array}$ \\
\hline 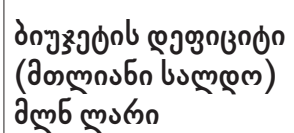 & -99 & -396 & -663 & -380 & -578 & 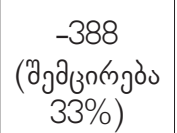 & $\begin{array}{l}-975 \\
(8 \text { roo } \\
151 \%)\end{array}$ \\
\hline
\end{tabular}




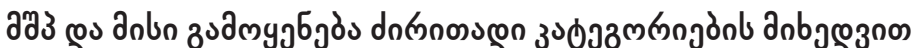

зbmomo 4 (www.geostat.ge)

\begin{tabular}{|c|c|c|c|c|c|c|c|c|}
\hline 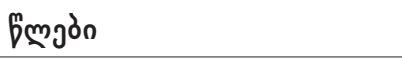 & 2010 & 2011 & 2012 & 2013 & 2014 & 2015 & 2016 & $2017^{*}$ \\
\hline 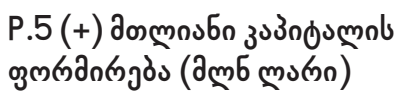 & 4477,7 & 6368 & 7575,4 & 6652,9 & 8688,9 & 10004,3 & 11136,6 & 12133,3 \\
\hline 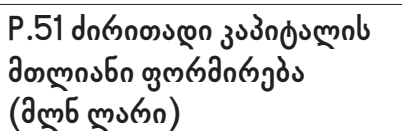 & 4009 & 5474,3 & 6496,8 & 5892,7 & 7534,7 & 9032 & 10310,3 & 11238,3 \\
\hline
\end{tabular}

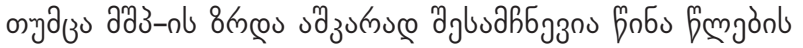

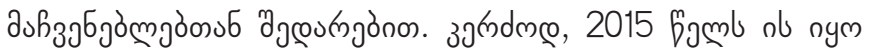

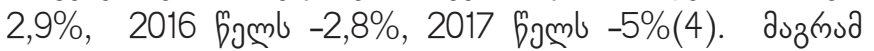

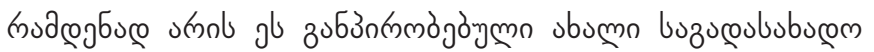

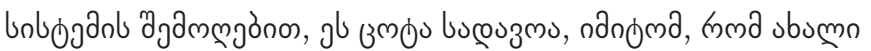

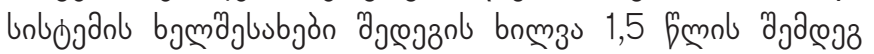

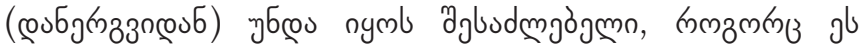

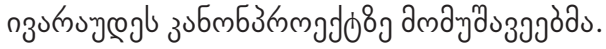

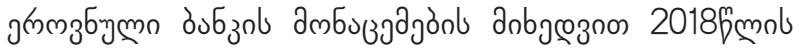

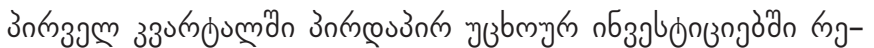

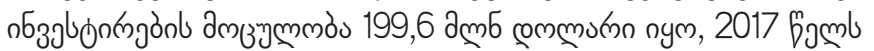

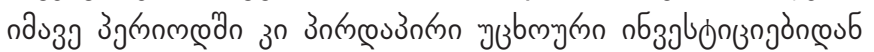

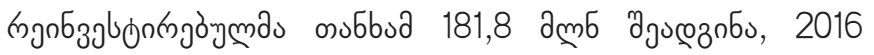

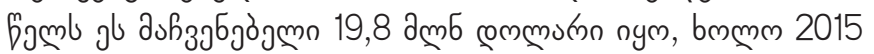

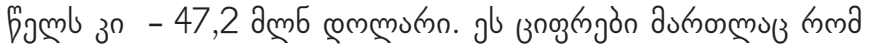

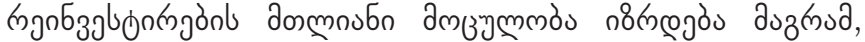

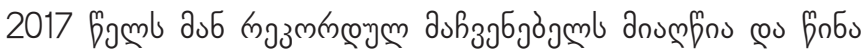
fmon auf

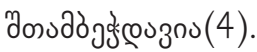

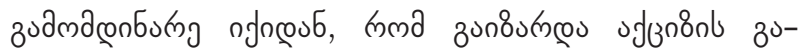

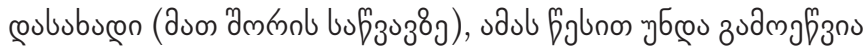

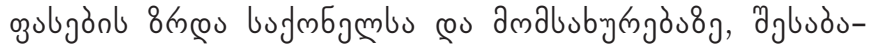

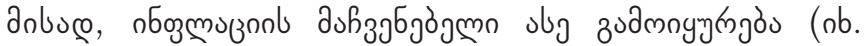

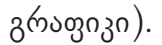

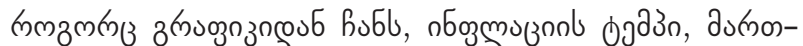

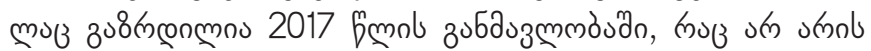

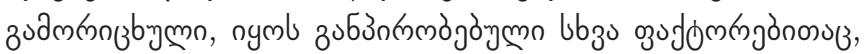

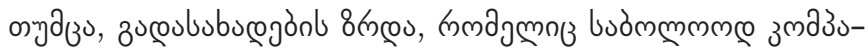

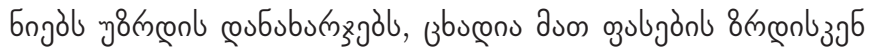

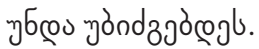

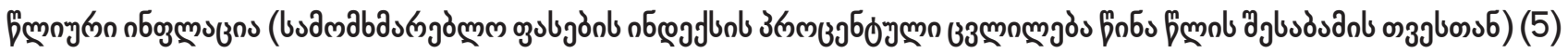

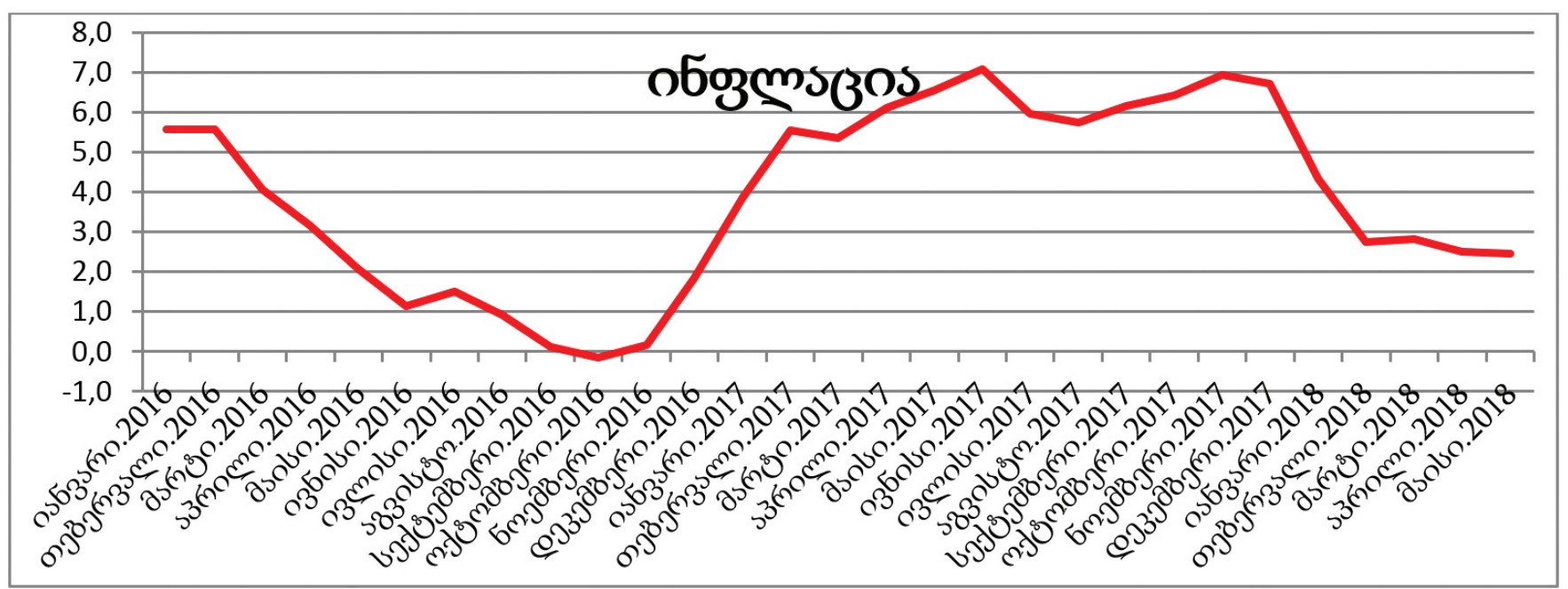

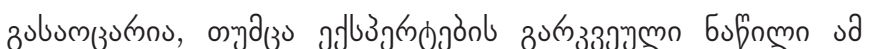

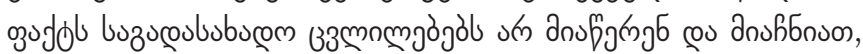

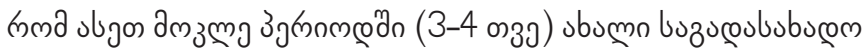

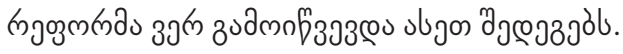

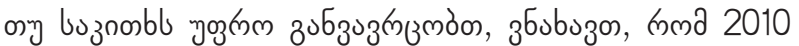

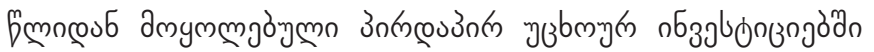

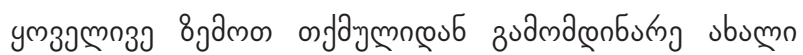

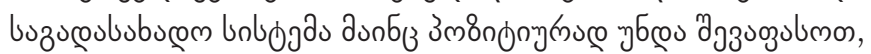

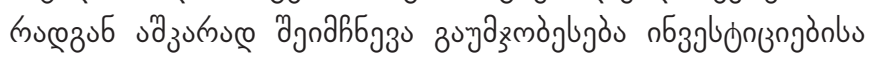

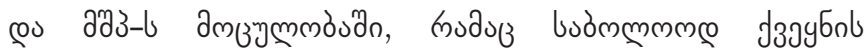

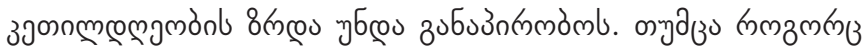

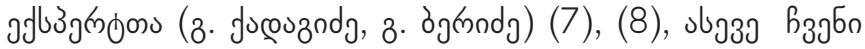




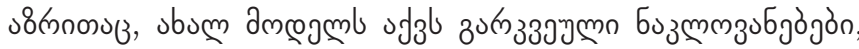

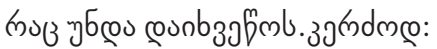

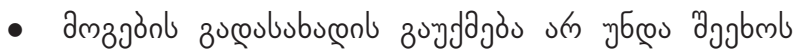

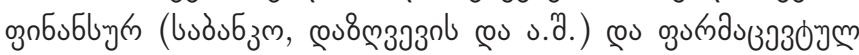

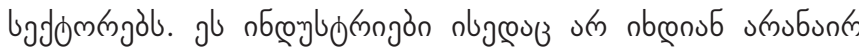

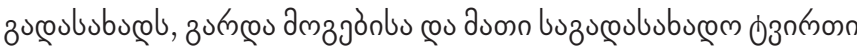

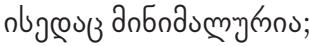

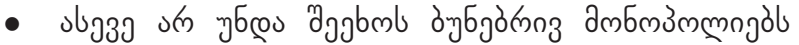

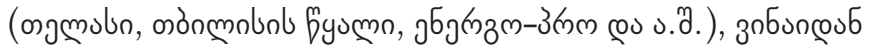

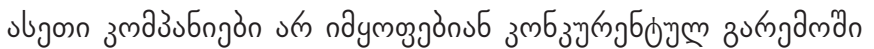

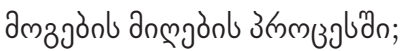

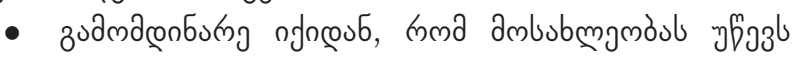

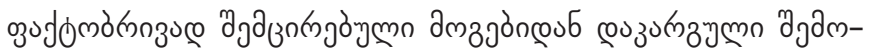

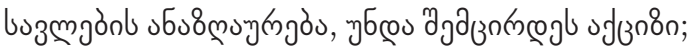

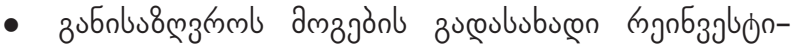

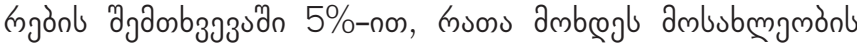

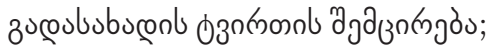

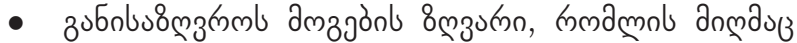

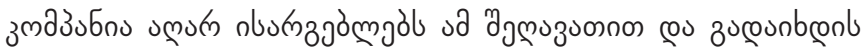
amsjöbl 15\%-b;

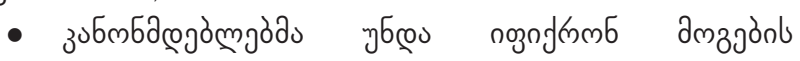

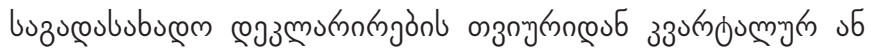

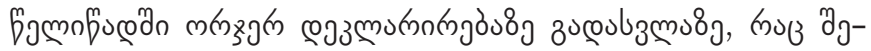

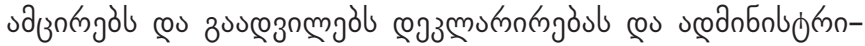
mgoubori;

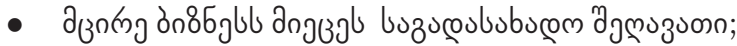

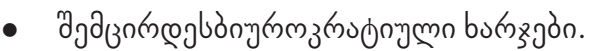

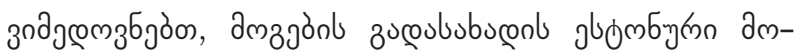

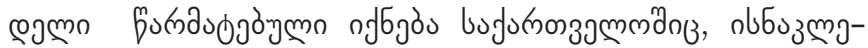

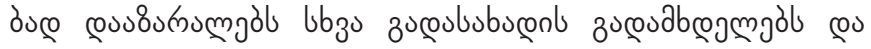
donmnubuco dmbsbmgmósl.

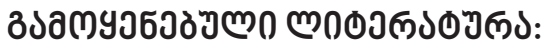

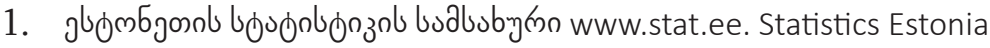

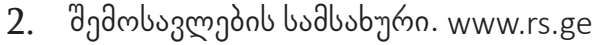

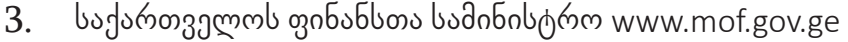

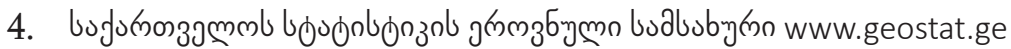

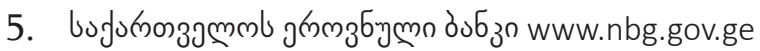

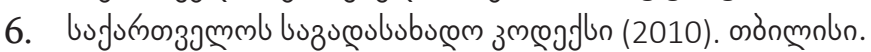

7. https://sputnik-georgia.com/economy/20161204/234058705/giorgi-qadagidze-khelisuflebas-rekomendaciebs-azlevs.html

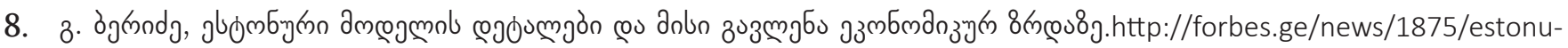
ri-modelis-detalebi-da-misi-gavlena-ekonomikur-zrdaze. 


\section{ESTONIAN MODEL OF PROFIT TAXAND ITS RESULTS IN GEORGIA}

\section{GULNAZ ERKOMAISHVILI}

https://doi.org/10.35945/gb.2018.06.023

Academic Doctor of Economics, Associate Professor

Ivane Javakhishvili Tbilisi State University, Georgia

gulnazi.erkomaishvili@tsu.ge

\section{DAVID ALUDAURI}

Ivane Javakhishvili Tbilisi State University, Georgia

davit.aludauri@gmail.com

\section{KEYWORDS: INCOME TAX, ESTONIAN MODEL, DISTRIBUTED PROFIT, TAX REFORM}

\section{SUMMARY}

The paper deals with one of the important legislative amendments in the Tax Code of Georgia, according to which the existing model of taxation was changed by the Estonian model. It was created in the agreement with Georgian legislation and with regard to Georgia's socio-economic condition. The purpose of this model is to accelerate economic growth and create favorable conditions for starting and developing business.

Based on the analysis of statistical data, the new tax system can be positively evaluated, as there is clearly visible improvement in the volume of investment and GDP, which will ultimately result in a country's well-being. 